\title{
Implication of BIS in the Migration and Invasion of A549 Non-small Cell Lung Cancer Cells
}

\author{
JEEHAN LEE ${ }^{1,2,3 *}$, HYE HYEON YUN ${ }^{1,2^{*}}$, SEULKI KIM ${ }^{3,4}$, SANG HEE JI ${ }^{1}$, \\ HYO-JEONG KUH ${ }^{4}$ and JEONG-HWA LEE ${ }^{1,2}$ \\ ${ }^{1}$ Department of Biochemistry, ${ }^{2}$ Institute of Aging and Metabolic Diseases, \\ ${ }^{3}$ Department of Biomedicine \& Health Sciences, Graduate School, \\ ${ }^{4}$ Department of Medical Life Sciences, College of Medicine, \\ The Catholic University of Korea, Seoul, Republic of Korea
}

\begin{abstract}
Background/Aim: High expression of the Bcl-2interacting cell death suppressor (BIS), an anti-apoptotic protein, in various human cancers is linked to a poor outcome. The purpose of this study was to clarify whether $B I S$ is associated with the migration and invasive characteristics of A549 cells. Materials and Methods: BISknockout (KO) cells were prepared by the CRISPR/Cas 9 method. The aggressive behaviors of A549 cells were analyzed by wound healing and a transwell invasion assay as well as 3D spheroid culture. Results: BIS depletion resulted in significant inhibition of the migration and invasive potential of A549 cells which was accompanied by an increased ratio of $E$-cadherin/N-cadherin and a decrease in the mRNA levels of Zeb1, Snail, Slug and MMP-2. NF-kB activity was suppressed in BIS-KO A549 cells due to the decrease in p65 protein levels, but not in mRNA levels. Conclusion: BIS regulates cell invasion and the induction of the epithelial-mesenchymal transition (EMT) phenotype in A549 cells probably via the $\mathrm{NF-KB}$ signaling pathway.
\end{abstract}

Lung cancer is one of the leading causes of cancer death worldwide (1). Non-small cell lung cancer (NSLCL) accounts for $85-90 \%$ of lung cancers. Despite the advances in our understanding of genetic alterations that are associated with the pathogenesis and resistance to conventional therapy

\footnotetext{
*These Authors contributed equally to this work.

Correspondence to: Jeong-Hwa Lee, Department of Biochemistry, College of Medicine, The Catholic University of Korea, Seoul 06591, Republic of Korea. Tel: +82 222587293, e-mail: leejh@catholic.ac.kr; Hyo-Jeong Kuh, Department of Medical Life Science, College of Medicine, The Catholic University of Korea, Seoul 06591, Republic of Korea. E-mail: hkuh@catholic.ac.kr
}

Key Words: BIS, A549, migration, invasion, EMT, NF-кB. which largely focuses on activating mutations of the epidermal growth factor receptor as well as anaplastic lymphoma kinase, the 5-year survival rate for all stages is around $17 \%$ (2). Thus, there is an urgent need to define the critical molecular events responsible for such poor patient outcomes, which are associated with therapeutic resistance as well as with distant metastasis.

Bcl-2-interacting cell death suppressor (BIS), also referred to as BAG3, has been shown to interact with various proteins including BCL-2, HSP70 or HSPB8, affecting various cellular processes including apoptosis, stress responses, autophagy or cytoskeleton arrangement (3-5). Consistent with the pro-survival activity of BIS against various types of apoptotic stress that has been shown in in vitro experiments, BIS is highly expressed in most human cancers $(6,7)$. Furthermore, a higher expression of BIS was shown to be associated with more aggressive phenotypes or a poorer prognosis in many types of human cancers including pancreatic cancer, ovarian cancer, glioblastomas, melanomas and hepatomas (8-12). Supporting the positive correlation between the degree of expression of BIS and clinical stages in several cancers, the migration and invasion abilities were profoundly affected by the modulation of BIS expression in various tumor cell lines derived from ovarian cancer, colorectal cancer, cervical cancer or hepatoma $(9,11,13$, 14). These findings raised the potential for BIS as a prognostic factor as well as a therapeutic target for a diverse list of human cancers. The possibility of a therapeutic target has increased in recent attempts to use BIS siRNAs that have been effectively transferred to glioblastoma and squamous cancer mouse models $(15,16)$. However, little is known regarding the role of BIS in NSCLC, especially with regard to migration and invasion.

In a previous study, we examined BIS expression in surgically resected NSCLC tissues, and the results indicated that BIS expression is increased in most squamous cell carcinomas and $70 \%$ of adenocarcinomas (17). In addition, an enhanced 
BIS expression was frequently detected at the periphery of a tumor tissue facing the adjacent nontumor region in adenocarcinomas, suggesting that BIS might contribute to the invasion of adenocarcinoma cells into normal tissues. In this report, therefore, we used A549 lung adenocarcinoma cell lines to access the biological impact of BIS on the migration or invasion of lung cancer cells. Our results indicate that depletion of BIS expression significantly suppressed A549 cell migration and invasion in vitro, and this was accompanied by decrease in MMP-2, snail and slug transcript levels. In addition, we present the possibility that NF-kB may function as an effector molecule that determines epithelial-mesenchymal transition (EMT) phenotype according to the amount of BIS.

\section{Materials and Methods}

Cell culture and transfection. Human non-small cell lung cancer cell (NSCLC) line A549 was provided by the American Type Culture Collection and maintained in RPMI 1640 medium supplemented with $10 \%$ fetal bovine serum (FBS, Biowest, Nuaillé, France) and 1\% penicillin-streptomycin (Biowest). BIS- knockout (KO) A549 cells were prepared using a CRISPR/Cas9 system, as described previously (18). Knock down of p65 proteins was carried out with small interfering RNA (siRNA) strategy using Lipofectamine 2000 reagent (Thermo Fisher Scientific, Waltham, MA, USA). Target specific sequences of siRNA were provided from Genolution (Seoul, Korea); siRNA for p65 (5'-CGGAUUGAGGAGAAACGUAAAUU-3') and control siRNA (5'-CCUACGCCACCAAUUUCGU-3').

Cell migration and invasion assay. Wound healing migration assay was performed on wild type (WT) and BIS-KO A549 cells. Cells were seeded in a $35 \mathrm{~mm}$ dish and grown overnight to confluence. The monolayer cells were scratched with the $200 \mu$ pipette tip to make a wound, and cells were washed with RPMI 1640 containing $10 \%$ FBS (Biowest) to remove dislodged cells, and then captured by an inverted microscope (Olymus, Center Valley, PA, USA). After $48 \mathrm{~h}$ of incubation, the cells migrating from the leading edge were captured again. The migrated cells were counted in each well and the mean values were presented after three independent experiments. For p65-siRNA-transfected A549 cells, transwell migration assay was performed in modified Boyden chambers with $8 \mu \mathrm{m}$ pore filter inserts in 24-well plates (Corning Life Sciences, Corning, NY, USA). $5 \times 10^{4}$ cells in $200 \mu$ l of serum free-RPMI 1640 medium were transferred to the upper chamber. The lower chamber was filled with RPMI 1640 containing 10\% FBS. After $24 \mathrm{~h}$ of incubation, the membrane was gently removed from the chamber, and the cells on the upper surface were removed using cotton swabs. Cells on the lower surface that pass through the filters were fixed with $50 \%$ methyl alcohol and stained with $0.1 \%$ crystal violet. The membranes were captured with digital images and the numbers of cells passed through the filters were counted in four random fields. The invasion ability was measured using Matrigel invasion chamber with $8 \mu \mathrm{m}$ pore (Corning Life Sciences) with the same procedures described for migration assay except the pre-hydration of membrane with $200 \mu \mathrm{l}$ of serum free RPMI 1640 medium.

Three-dimensional (3D) spheroid culture in microchannel plates. Microchannel plates were prepared using polydimethylsiloxane
(PDMS; Silgard 184, Dow Chemical, Midland, MI, USA) according to the protocol reported in previous studies $(19,20)$. Cell-hydrogel mixture was prepared at a cell density of $5 \times 10^{5} / \mathrm{ml}$ in $2.33 \mathrm{mg} / \mathrm{ml}$ type I collagen solution (BD Biosciences, San Jose, CA, USA) containing phenol red with $\mathrm{pH}$ adjusted to 7.4. Three $\mu$ l of cell-hydrogel mixture was loaded into designated cell channels and pre-incubated for $30 \mathrm{~min}$ allowing polymerization before adding media. Spheroid cultures were monitored with media changes on a daily basis. At day 6 , cells were stained for F-actin and nucleus using rhodamine phalloidin (1:1,000, cat. no. R415, Thermo Fisher) and DAPI (1:1,000, cat. no. D9564, Sigma-Aldrich, St. Louis, MO, USA), respectively. The cells in the media channels were identified as migrated cells out of cell channels and counted on the images acquired under confocal microscopy (LSM 800 Meta, Zeiss, Oberkochen, Germany).

Western blot analysis and co-immunoprecipitation. Western blot analysis was performed as previously described. The primary antibodies used in this study are anti-BIS antibody (3), anti $\mathrm{N}$ cadherin, anti E-cadherin, anti-IkB (Santa Cruz-Biotechnology, Santa Cruz, CA, USA), anti-p65 (Cell Signaling, Danvers, MA, USA) and anti $\beta$-actin (Sigma -Aldrich). The interaction of BIS with p65 was validated by co-immunoprecipitation assay. Cells were lysed with RIPA buffer (50 mM Tris, $150 \mathrm{mM} \mathrm{NaCl}, 1 \% \mathrm{NP}-40,0.5 \%$ sodium deoxycholate, $0.1 \%$ sodium dodecyl sulfate, $\mathrm{pH} 7.5$ ) containing protease inhibitor and phosphatase inhibitor (Sigma Aldrich). After centrifugation at $15,000 \mathrm{~g}$ for $30 \mathrm{~min}$, each cell lysate was precleared by PureProteome ${ }^{\mathrm{TM}}$ protein A magnetic beads (Millipore, Billerica, MA, USA) for $2 \mathrm{~h}$ at $4^{\circ} \mathrm{C}$. And then, the specific anti-BIS serum or normal rabbit serum was added to $1 \mathrm{mg}$ of cell lysates and further incubated for overnight at $4^{\circ} \mathrm{C}$. Next day, the pre-equilibrated beads were added to cell lysates and incubated for $4 \mathrm{~h}$ at $4^{\circ} \mathrm{C}$ while rocking. After washing with lysis buffer three times, beads were resuspended with 2X Laemmli buffer. BIS or p65 expression in immunoprecipitates was analyzed by western blot analysis.

Quantitative real time RT-PCR ( $q R T-P C R)$. RNA was isolated using RNAiso Plus (TaKara Biotechnology, Shiga, Japan) and cDNA was synthesized by AccuPower ${ }^{\circledR}$ Customized RocketScript ${ }^{\mathrm{TM}}$ Cycle RT premix (Bioneer, Daejeon, Korea). RNA expression levels were measured by standard qPCR procedures with SYBR Premix Ex Taq ${ }^{\mathrm{TM}}$ (Takara Biotechnology) on Applied Biosystems 7300 machine (Applied Biosystems, Carlsbad, CA, USA). The relative values for specific mRNA were yielded after normalization to the $\mathrm{Ct}$ value from $\beta$-actin in the same sample using the ddCt method. The primers list are shown in Table I.

$N F-k B$ luciferase reporter assay. The NF-kB luciferase reporter assay was performed as described previously (21). Cells were transfected with a reporter plasmid containing a $\mathrm{kB}$ response element (IgkB-Luc, $0.5 \mu \mathrm{g})$ and a renilla reporter plasmid $(0.005 \mu \mathrm{g})$ with Fugene 6 (Roche Life Science, Basel, Switzerland) for $24 \mathrm{~h}$. The reporter activity was determined by a dual luciferase reporter gene assay kit (Promega, Madison, WI, USA). After normalizationwith renilla activity, the NF-kB activity as a transcriptional activator in BIS-KO A549 cells was expressed relatively to that in WT A549 cells.

Statistics. All of the experiments were performed at least three times and the results are expressed as mean \pm standard deviation (SD). Student's $t$-test was used to compare data between two groups. $p$-Value of less than 0.05 was considered statistically significant. 
Table I. Specific primers used in this study.

\begin{tabular}{|c|c|c|}
\hline Target gene & Forward & Reverse \\
\hline Zebl & 5'-GCCAATAAGCAAACGATTCTG-3' & 5'-TTTGGCTGGATCACTTTCAAG-3' \\
\hline Snail & 5'-ССТCССТGTCAGATGAGGAC-3' & 5'-CCAGGCTGAGGTATTCCTTG-3' \\
\hline Slug & 5'-GGGGAGAAGCCTTTTTCTTG-3' & 5'-TCCTCATGTTTGTGCAGGAG-3' \\
\hline$M M P 2$ & 5'-GCAAGGAGTACAACAGCTGC-3' & 5'-GAAGCGGAATGGAAACTTGCA-3' \\
\hline p65 & 5'-CCСATCTTTGACAATCGTGC-3' & 5'-ATCAGCTTGCGAAAAGGAGC-3' \\
\hline$\beta$-Actin & 5'-AGTACTCCGTGTGGATCGGC-3' & 5'-GCTGATCCACATCTGCTGGA-3' \\
\hline
\end{tabular}

\section{Results}

Migration and invasive potential of A549 cells was suppressed by BIS depletion in vitro. In a previous study, we observed that BIS expression was higher in the marginal area compared to central region in adenocarcinoma tissues (17). To determine if high expression of BIS is associated with the aggressive phenotype of adenocarcinoma of lung origin, we examined the effect of BIS depletion on the migration and invasiveness of A549 cell line derived from a pulmonary adenocarcinoma using BIS-KO A549 cells prepared by CRISPR/Cas9 (18). As shown in Figure 1A, BIS-KO A549 cells revealed a significant decrease in the wound healing capacity of A549 cells to about $55.5 \%$ of that of WT A549 cells $(p=0.06)$. The complete depletion of BIS also inhibited the invasive ability of A549 cells as evidenced by a definite reduction in the number of cells that invaded through a matrigel-coated membrane, $170 \pm 12$ and $36 \pm 9$ cells for WT and BIS-KO A549 cells, respectively (Figure $1 \mathrm{~B}, p<0.001$ ). Furthermore, the functional role of BIS in the infiltrative behavior of A549 cells was evaluated under a physiologically relevant condition using microchannel culture chips. Both types of cells were cultured in collagen-incorporated microfluidic channels and their migration out of the channels was then monitored. Under these conditions, cells were grown as 3D spheroids that provide in vivo-like $3 \mathrm{D}$ cell-cell and cell-extracellular matrix interaction (20). Over the 6 days of culture, both WT and BIS-KO A549 cells formed discrete tumor spheroids with no significant difference in the number or size of tumor spheroids (Figure 2A). However, the migration of BIS-KO A549 cells out of the spheroid channel was substantially suppressed compared to WT A549 cells, as determined by cell numbers and the distance that they moved out of the channel (Figure 2B). The total number of migrated cells was 158.0 and 41.25 for WT A549 and BIS-KO A549 cells, respectively $(p<0.05)$. The average distance moved was $151.5 \mu \mathrm{m}$ in the case of WT A549 cells, while the value was $108.9 \mu \mathrm{m}$ in BIS-KO A549 cells $(p=0.068)$. Thus, BIS depletion significantly inhibited the migration and invasion of A549 cells, not only in 2D cultures but also in collagen-supported 3D conditions which more closely represents in vivo conditions.
BIS depletion induces a reversion of EMT in A549 cells. EMT is an important process which provides the phenotypic switch that causes tumor cells to acquire more aggressive properties (22). The expression of E-cadherin, an epithelial marker and $\mathrm{N}$-cadherin, a mesenchymal marker, were therefore examined in WT and BIS-KO A549 cells. Western blotting indicated that E-cadherin levels were increased while those for N-cadherin were decreased in BIS-KO A549 cells compared to WT A549 cells (Figure 3A). Also, the expressions of several EMT-inducing transcription factors such as Zeb1, Snail1 and Slug were notably inhibited by BIS depletion, as determined by qRT-PCR (Figure 3B). In addition, MMP-2 expression was also substantially decreased in BIS-KO A549 compared to WT A549 cells, consistent with the reduction in the invasive ability of BIS-KO A549 cells. These collective results indicate that the retarded migration and invasion behavior of A549 cells upon BIS depletion is mediated by, at least partly, reversing EMT program in A549 cells.

$N F-k B$ signaling is involved in BIS-mediated regulation of migration and invasive potential of A549 cells. Previous studies have suggested that NF-KB plays an essential role in the induction of EMT in several types of tumor cells including A549 cells (23-25). On the other hand, it has been shown that $\mathrm{BIS}$ is required for the sustained increase in NF-kB signaling in several tumor cells through the inhibition of HSP70dependent degradation of $\operatorname{IKK} \gamma$ (26). In addition, BIS knockdown decreased the translocation of p65 to the nucleus upon oxidative stress (27). To investigate the association of $\mathrm{NF}-\mathrm{kB}$ in BIS-mediated EMT, we first compared the NF- $\mathrm{BB}$ activity in WT A549 and BIS KO A549 cells using a NF-kB reporter construct. Figure 4A shows that the luciferase activity driven from NF-kB response element was significantly lower in BIS-KO A549 cells, 55\% of that in WT A549 cells $(p<0.001)$. We then examined whether BIS depletion affects the expression levels of p65 or IkB. As shown in Figure 4B, while the IkB levels were not different between the two cells, p65 protein levels were decreased in BIS-KO A549 cells to approximately $56.4 \%$ of that in WT A549 cells $(p<0.01)$. Subsequent analyses for p65 mRNA levels indicated that there 

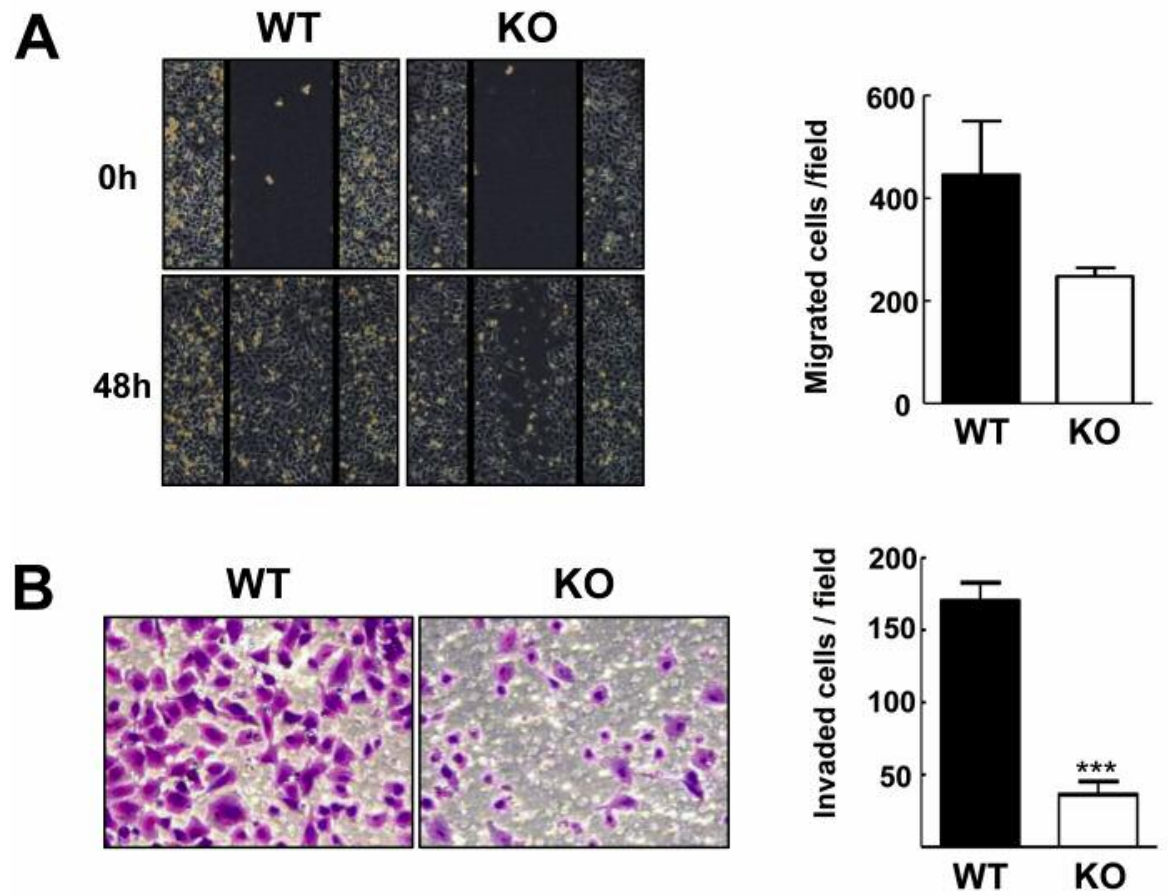

Figure 1. Effect of BIS depletion on the migration and invasion of A549 cells. (A) The migration behavior of WT and BIS-KO A549 cells was assessed by means of a wound healing assay. Representative images of the wound area were obtained by phase-contrast microscopy at 0 and $48 \mathrm{~h}$ post-scratching (left). The average number of migrated cells from triplicate experiments is summarized in the right panel. (B) Transwell invasion assay shows that BIS depletion inhibited the invasion of A549 cells (left). The results from three independent experiments are provided (right). Data are presented as the mean $\pm S D$. $* * * p<0.001 \mathrm{vs}$. WT.

A

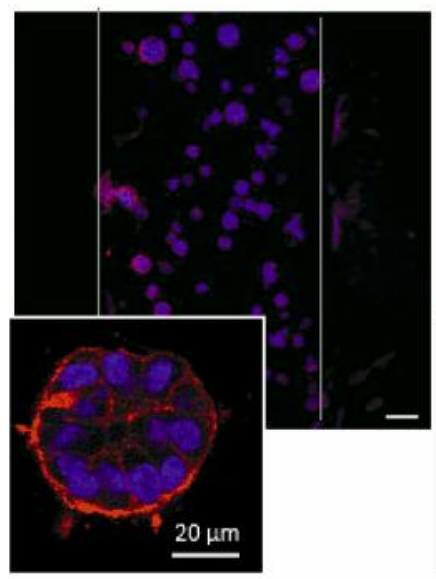

Figure 2. Decreased migration of A549 cells out of spheroid channels by BIS depletion. (A) WT and BIS-KO A549 cells were cultured in collagen-supported microfluidic channels for 6 days. Spheroid formation was shown by staining the nuclei (DAPI: blue) and F-actin (red). Magnified view of a spheroid is shown in the lower panel. Scale bar, $100 \mu m$. (B) Representative images of WT and BIS-KO A549 cells migrated out of the spheroid channel into the media channel (upper). Scale bar, $50 \mu \mathrm{m}$. The total number of cells found in the media channel and the distance moved was determined from four independent experiments and expressed as the mean $\pm S D$ (lower). ${ }^{*} p<0.05 v s$. WT.
B
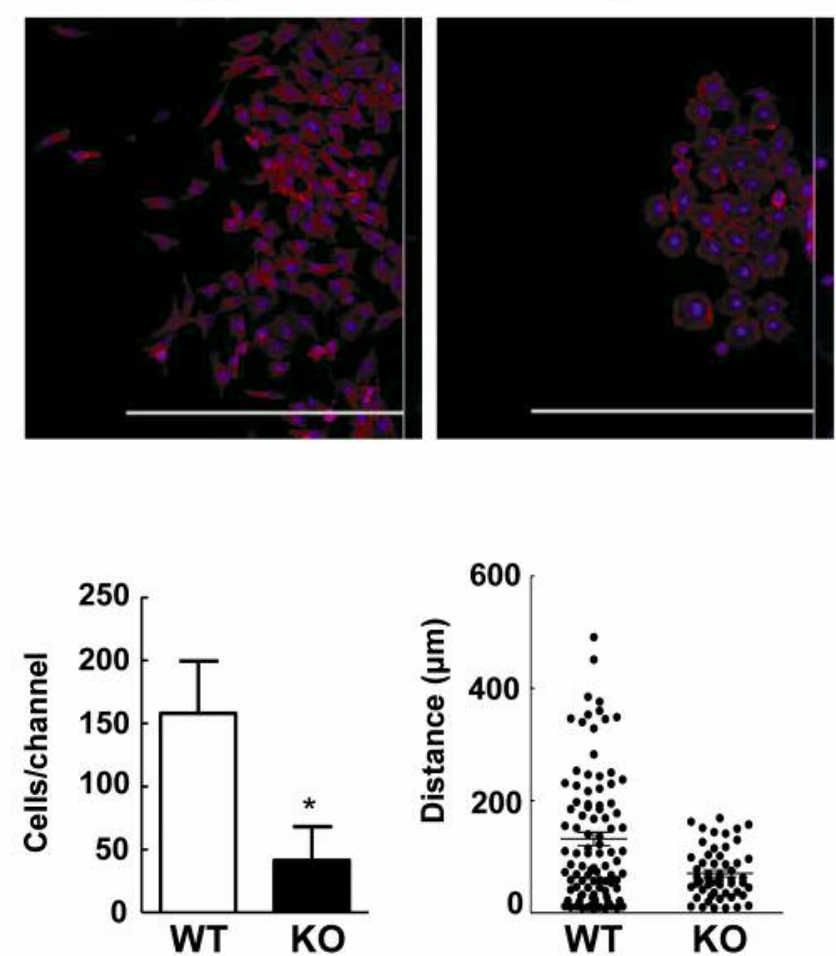

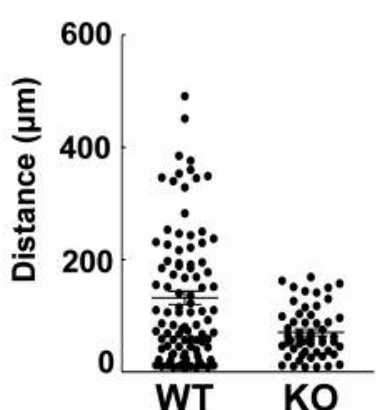


A

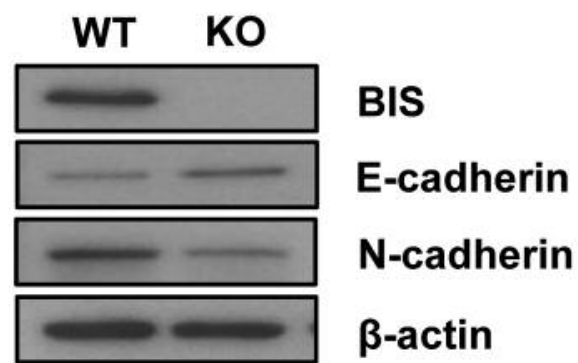

B

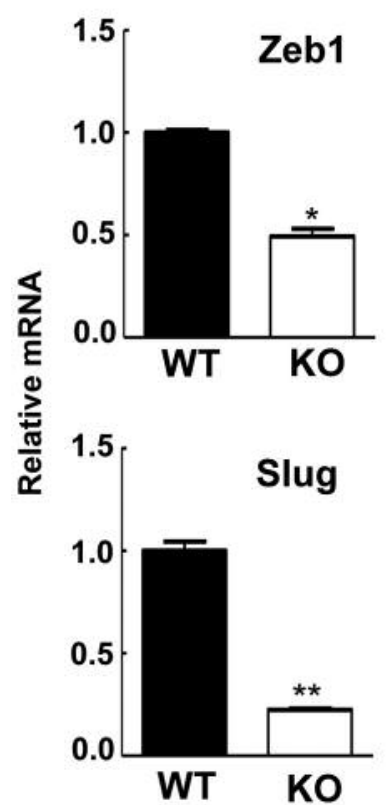

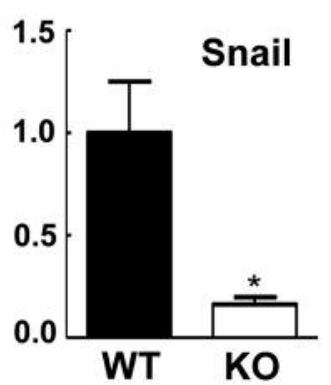

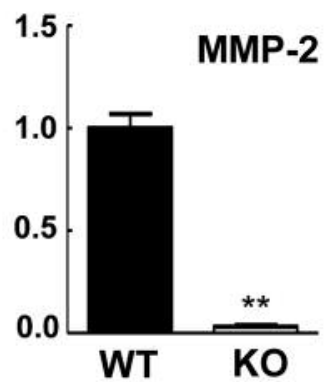

Figure 3. Expression of EMT markers in WT and BIS-KO A549 cells. (A) Western blotting assay for expression of BIS, E-cadherin and $N$ cadherin in WT and BIS-KO A549 cells. Beta-actin expression was provided as loading control. (B) The relative $m R N A$ expressions of EMT-related factors were determined by qRT-PCR. Data are expressed as the mean $\pm S D$. $* p<0.05, * * p<0.01$ vs. WT.

was no significant decrease in p65 mRNA levels in BIS-KO A549 cells compared to WT A549 cells (Figure 4C). These findings suggest that BIS is required for NF-kB signaling, modulating the stability of the p65 protein rather than transcriptional regulation. To confirm this thesis, the rate of degradation of p65 was determined by cycloheximide (CHX) chase experiments. Figure 4D shows that p65 levels were degraded more rapidly in BIS-KO A549 cells than in WT A549 cells. A co-immunoprecipitation assay revealed that p65 was detected in the immune complex which was precipitated by anti-BIS, indicating that BIS is physically associated with

p65 in A549 cells (Figure 4E). Finally, we confirmed that the migration and invasion of A549 cells can be reduced sufficiently by the knockdown of p65 itself (Figure 5).

Taken together, our results suggest that BIS is required for stabilization of p65 protein through their protein-protein interactions, leading to the constitutive activation of NF-kB. And the BIS-dependent NF-kB activation might contribute, at least in part, to sustaining the migration and invasion capabilities of A549 cells.

\section{Discussion}

Although conflicting results have been reported, BIS has been reported to promote the migration and invasion in many types of tumor cells $(9,13,28)$. In the present study, the complete depletion of BIS using a CRISPR/Cas9-mediated knockout strategy was found to effectively inhibit the migration and invasion in A549 cells via reversing the EMT process. This is consistent with our previous report showing higher levels of BIS expression in the marginal area adjacent to normal tissue than central area in human lung cancer tissues (17). Therefore, our results reaffirm that BIS appears to be an important molecule that can regulate cell survival, as well as invasive properties of various types of cancer including lung adenocarcinoma, which is linked to clinically unfavorable outcomes.

Several reports have suggested EMT regulation as a mechanism that promotes the development of aggressive phenotype by BIS $(11,29,30)$. However, most previous reports only reported changes in the EMT phenomena as a result of quantitative changes in BIS, and specific details on how BIS can affect EMT have not been elucidated. Of the several pathways that initiate EMT in tumor cells, a possible link was noted between NF-kB activity and BIS-mediated EMT in A549 cells, by demonstrating a significant decrease in NF-kB activity which can be attributed to a decrease in p65 levels in BIS-KO A549 cells. Furthermore, it was also observed that the knockdown of p65 resulted in the suppression of migration and invasion ability of A549 cells. These results are consistent with previous reports indicating that the activation of NF-kB is involved in the EMT process in A549 cells in response to several stimuli including TGF$\beta$, acetylcholine and the cell cycle activator $\operatorname{RGC} 32(24,31$, 32). In addition, it has been reported that the EMT of A549 cells was markedly inhibited by certain conditions that regulate NF-kB activity such as SIRT1 overexpression as well as Osthole, a naturally-occurring coumarin derivative (24 33). Based on these results, BIS targeting could also be an effective therapeutic modality for controlling EMT mediated by NF-kB and subsequent metastasis in NSCLC.

Since the BIS protein has no enzymatic activity, the diverse effects of BIS on the fate of cells can be attributed to its interaction with various proteins, not to the BIS protein itself, thus affecting their stability, localization and activity (4). For 


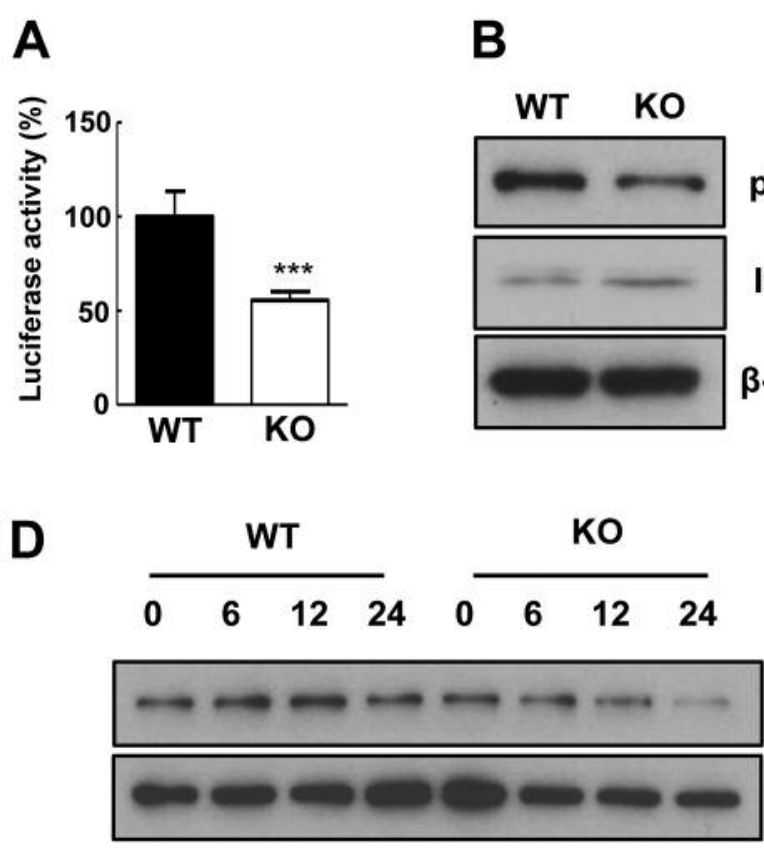

(h)

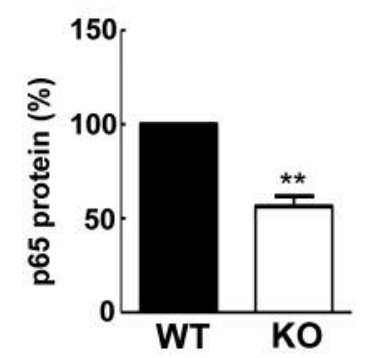

C

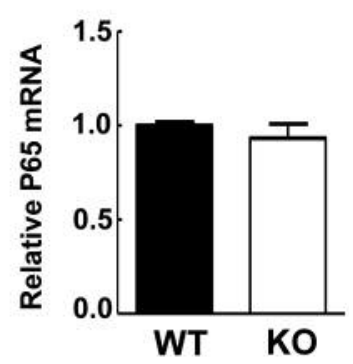

p65

$\beta$-actin
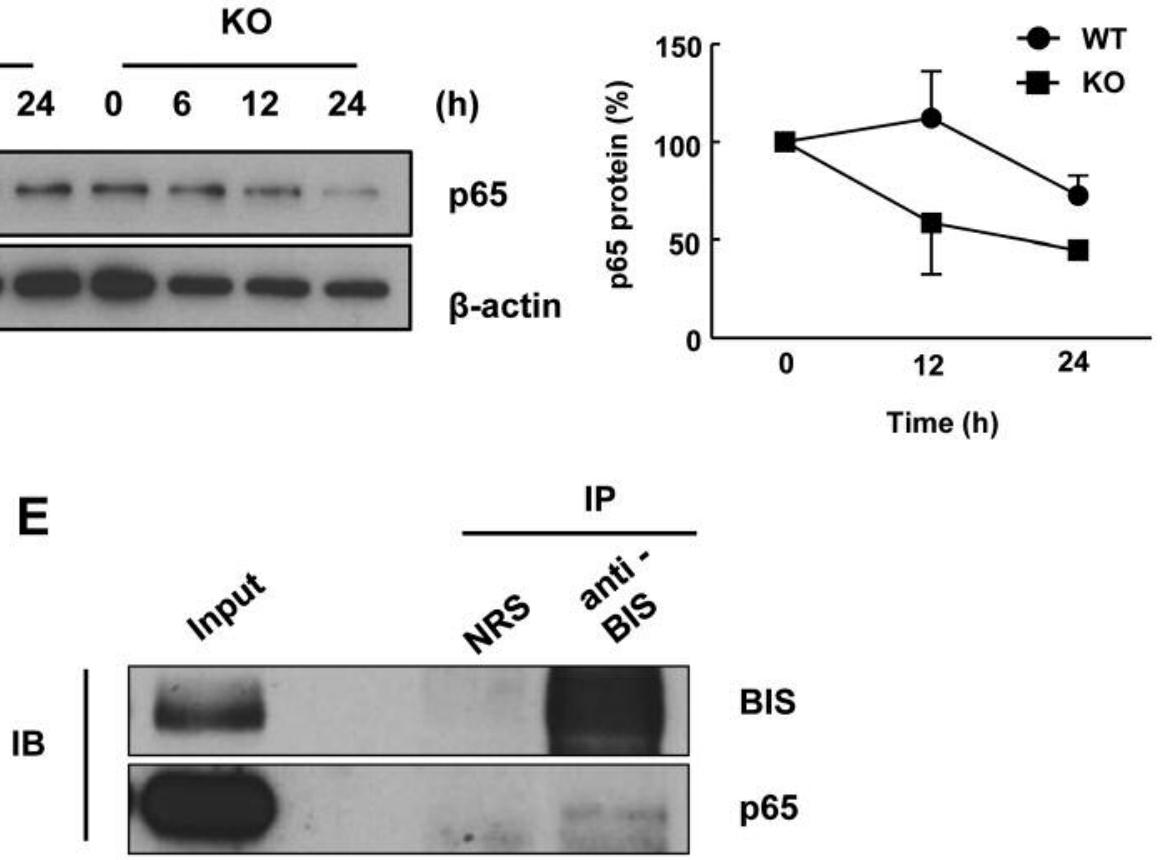

Figure 4. NF-KB signaling is suppressed in BIS-KO A549 cells. (A) NF-xB activity was measured by a luciferase reporter assay. The relative value is shown as a percentage of that in WT A549 cells. (B) Western blotting for p65 and IKB in WT and BIS-KO A549 cells was shown (left). The quantitative data for western blot of p65 is shown (right). (C) The relative p65 mRNA levels were determined by qRT-PCR in WT and BIS-KO A549 cells. (D) Cycloheximide $(20 \mu \mathrm{g} / \mathrm{ml})$ was added to A549 cells for the indicated times and p65 expression was determined by western blotting (left). The quantitative results from three independent experiments were compared between WT and BIS-KO A549 cells (right). (E) The interaction of BIS and p65 was demonstrated by co-immunoprecipitation (IP) with ant-BIS serum followed by immuno blotting (IB) with anti-p65 antibody. NRS: Normal rabbit serum. ${ }^{* *} p<0.01,{ }^{* * *} p<0.001 \mathrm{vs}$. WT.

example, the representative anti-apoptotic protein Mcl-1 is stabilized by its interaction with BIS, which prevents it from undergoing HSP70-dependent degradation (34). On the other hand, the HSP70/BIS complex is able to prevent the translocation of BAX to mitochondria (10). In the present study, we demonstrated that BIS interacts with p65 and that p65 protein stability was decreased by BIS depletion as evidenced by co-immunoprecipitation and $\mathrm{CHX}$ chase analysis, respectively (Figure 5). Considering that HSP70 was shown to mediate the degradation of the p65 in some types of immune cells $(35,36)$, it is possible that an interaction of BIS and p65 might relieve p65 from HSP70-dependent degradation, as previously suggested for the availability of IKK $\gamma$, which was increased following interaction with BIS while decreased by interaction with HSP70 (26). Thus, a high expression of BIS might confer stabilization of $\mathrm{p} 65$ which sustains NF-kB activity in tumor cells, including NSCLC, which might contribute, at least partly, to the subsequent maintenance of EMT phenotypes as well as enhanced cell survival. The association of BIS and p65, however, was also shown to suppress p65 binding to the $\mathrm{kB}$ motif of the HIV-1 promoter in glial cells (37). Therefore, BIS appears to exert both stimulatory and inhibitory effects on 
A
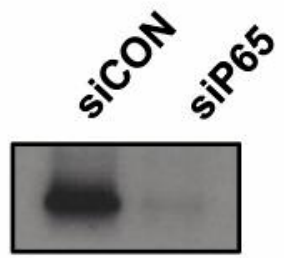

p65

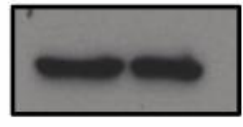

\section{$\beta$-actin}

B
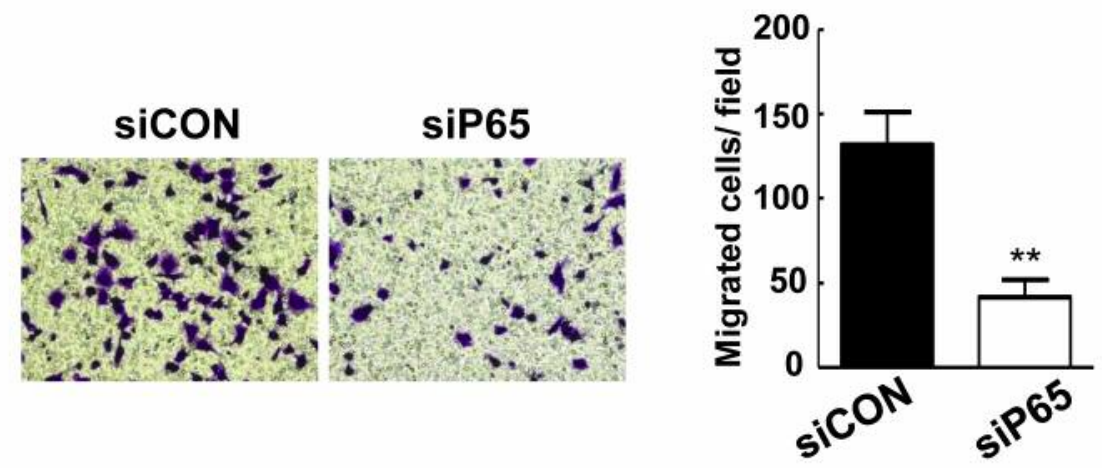

C
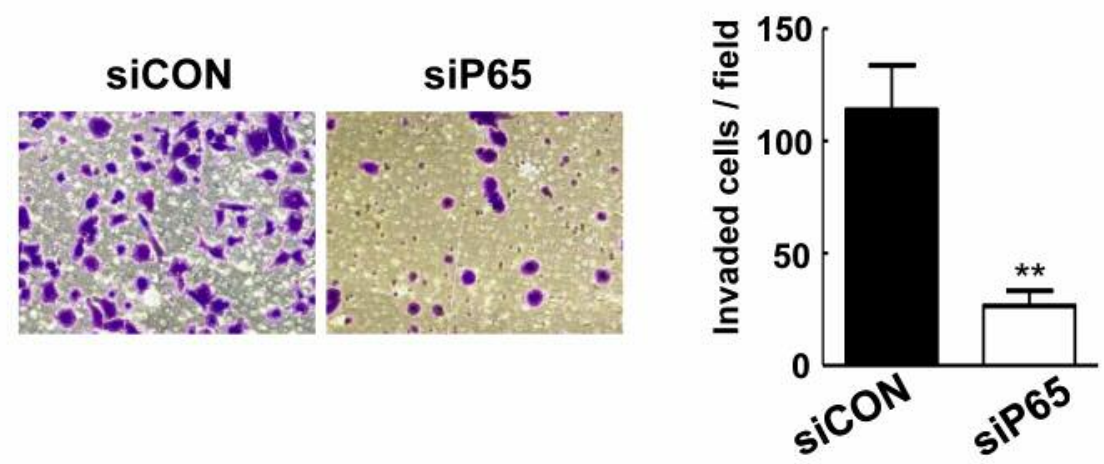

Figure 5. Effect of knockdown of p65 on the migration and invasion of A549 cells. (A) Western blotting showing the effective suppression of p65 after the transfection of control (siCON) and p65 specific siRNA (siP65). (B, C) Silencing of p65 inhibited the migration (B) and invasion (C) of A549 cells as determined by a transwell assay (left). The quantitative results are shown in right panels. Data are expressed as the mean $\pm S D$. $* * p<0.01$ vs. siCON.

NF-kB signaling depending on the cell types as well as the conditions under which the cells are in, such as whether or not various stressors or activators are present. In the present and previous studies (38), BIS-KO A549 cells exerted distinct characteristics on the migration and invasive ability, as well as their sensitivity to anti-cancer drugs. Therefore, BIS-KO A549 cells could be used as an important tool to develop strategies to efficiently control the proliferation and migration of NSCLC simultaneously with depletion of BIS.

In conclusion, BIS regulates cell invasion and the induction of EMT phenotype in lung cancer cells, probably in association with the NF-kB signaling pathway. Therefore, the BIS and NF-kB pathway may be a useful therapeutic target for suppressing the invasive potential of NSCLC.

\section{Conflicts of Interest}

The Authors declare no conflicts of interest regarding this study.

\section{Acknowledgements}

This research was supported by Basic Science Research Programs through the National Research Foundation of Korea (NRF), funded by the Minister of Education, Science and Technology (2012R1A5A2047939 and 2017R1A2B2005508). 


\section{References}

1 Jemal A, Center MM, DeSantis C and Ward EM: Global patterns of cancer incidence and mortality rates and trends. Cancer Epidemiol Biomarkers Prev 19: 1893-1907, 2010.

2 Rotow $\mathrm{J}$ and Bivona TG: Understanding and targeting resistance mechanisms in NSCLC. Nat Rev Cancer 17: 637-658, 2017.

3 Lee JH, Takahashi T, Yasuhara N, Inazawa J, Kamada S and Tsujimoto Y: Bis, a Bcl-2-binding protein that synergizes with $\mathrm{Bcl}-2$ in preventing cell death. Oncogene 18: 6183-6190, 1999.

4 Rosati A, Graziano V, De Laurenzi V, Pascale M and Turco MC: BAG3: a multifaceted protein that regulates major cell pathways. Cell Death Dis 2: e141, 2011.

5 Sturner E and Behl C: The role of the multifunctional BAG3 protein in cellular protein quality control and in disease. Front Mol Neurosci 10: 177, 2017.

6 De Marco M, Basile A, Iorio V, Festa M, Falco A, Ranieri B, Pascale M, Sala G, Remondelli P, Capunzo M, Firpo MA, Pezzilli R, Marzullo L, Cavallo P, De Laurenzi V, Turco MC and Rosati A: Role of BAG3 in cancer progression: A therapeutic opportunity. Semin Cell Dev Biol 78: 85-92, 2018.

7 Yunoki T, Tabuchi Y and Hayashi A: Expression of antiapoptotic protein BAG3 in human sebaceous gland carcinoma of the eyelid. Anticancer Res 37: 1931-1934, 2017.

8 Rosati A, Bersani S, Tavano F, Pozza ED, De Marco M, Palmieri M, De Laurenzi V, Franco R, Scognamiglio G, Palaia R, Fontana A, Di Sebastiano P, Donadelli M, Dando I, Medema JP, Dijk F, Welling L, di Mola FF, Pezzilli R, Turco MC and Scarpa A: Expression of the Antiapoptotic protein BAG3 is a feature of pancreatic adenocarcinoma and its overexpression is associated with poorer survival. Am J Pathol 181: 1524-1529, 2012.

9 Suzuki M, Iwasaki M, Sugio A, Hishiya A, Tanaka R, Endo T, Takayama S and Saito T: BAG3 (BCL2-associated athanogene 3) interacts with MMP-2 to positively regulate invasion by ovarian carcinoma cells. Cancer Lett 303: 65-71, 2011.

10 Festa M, Del Valle L, Khalili K, Franco R, Scognamiglio G, Graziano V, De Laurenzi V, Turco MC and Rosati A: BAG3 protein is overexpressed in human glioblastoma and is a potential target for therapy. Am J Pathol 178: 2504-2512, 2011.

11 Xiao H, Cheng S, Tong R, Lv Z, Ding C, Du C, Xie H, Zhou L, $\mathrm{Wu} \mathrm{J}$ and Zheng S: BAG3 regulates epithelial-mesenchymal transition and angiogenesis in human hepatocellular carcinoma. Lab Invest 94: 252-261, 2014.

12 Guerriero L, Chong K, Franco R, Rosati A, De Caro F, Capunzo $\mathrm{M}$, Turco MC and Hoon DS: BAG3 protein expression in melanoma metastatic lymph nodes correlates with patients' survival. Cell Death Dis 5: e1173, 2014.

13 Shi H, Xu H, Li Z, Zhen Y, Wang B, Huo S, Xiao R and Xu Z: BAG3 regulates cell proliferation, migration, and invasion in human colorectal cancer. Tumour Biol 37: 5591-5597, 2016.

14 Wang Y and Tian Y: miR-206 inhibits cell proliferation, migration, and invasion by targeting BAG3 in human cervical cancer. Oncol Res, 2018. doi: 10.3727/096504017X15143 731031009. [Epub ahead of print]

15 An S, He D, Wagner E and Jiang C: Peptide-like polymers exerting effective glioma-targeted siRNA delivery and release for therapeutic application. Small 11: 5142-5150, 2015.
16 Wang BK, Yu XF, Wang JH, Li ZB, Li PH, Wang H, Song L, Chu PK and Li C: Gold-nanorods-siRNA nanoplex for improved photothermal therapy by gene silencing. Biomaterials 78: 27-39, 2016.

17 Yeo CD, Park GS, Kang N, Choi SY, Kim HY, Lee DS, Kim YS, Kim YK, Park JG, Sung SW, Lee KY, Park MS, Yim HW, Kim $\mathrm{SJ}$ and Lee JH: Bis Expression in patients with surgically resected lung cancer and its clinical significance. Ann Surg Oncol 22: S1365-1370, 2015.

18 Yun HH, Baek JY, Seo G, Kim YS, Ko JH and Lee JH: Effect of BIS depletion on HSF1-dependent transcriptional activation in A549 non-small cell lung cancer cells. Korean J Physiol Pharmacol 22: 457-465, 2018.

19 Jeong SY, Lee JH, Shin Y, Chung S and Kuh HJ: Co-Culture of Tumor Spheroids and Fibroblasts in a Collagen MatrixIncorporated Microfluidic Chip Mimics Reciprocal Activation in Solid Tumor Microenvironment. PLoS One 11: e0159013, 2016.

20 Lee JH, Kim SK, Khawar LA, Jeong SY, Chung S and Kuh HJ: Microfluidic co-culture of pancreatic tumor spheroids with stellate cells as a novel 3D model for investigation of stroma-mediated cell motility and drug resistance. J Exp Clin Cancer Res 37: 4, 2018.

21 Byun MS, Jeon KI, Choi JW, Shim JY and Jue DM: Dual effect of oxidative stress on NF-kappakB activation in HeLa cells. Exp Mol Med 34: 332-339, 2002.

22 Tsai JH and Yang J: Epithelial-mesenchymal plasticity in carcinoma metastasis. Genes Dev 27: 2192-2206, 2013.

$23 \mathrm{Wu}$ Y and Zhou BP: TNF-alpha/NF-kappa B/Snail pathway in cancer cell migration and invasion. Brit J Cancer 102: 639-644, 2010.

24 Feng H, Lu JJ, Wang Y, Pei L and Chen X: Osthole inhibited TGF beta-induced epithelial-mesenchymal transition (EMT) by suppressing NF-kappaB mediated Snail activation in lung cancer A549 cells. Cell Adh Migr 11: 464-475, 2017.

25 Chen KC, Chen CY, Lin CR, Yang TY, Chen TH, Wu LC and Wu CC: Luteolin attenuates TGF-beta1-induced epithelialmesenchymal transition of lung cancer cells by interfering in the PI3K/Akt-NF-kappaB-Snail pathway. Life Sci 93: 924-933, 2013.

26 Ammirante M, Rosati A, Arra C, Basile A, Falco A, Festa M, Pascale M, d'Avenia M, Marzullo L, Belisario MA, De Marco M, Barbieri A, Giudice A, Chiappetta G, Vuttariello E, Monaco M, Bonelli P, Salvatore G, Di Benedetto M, Deshmane SL, Khalili K, Turco MC and Leone A: IKK \{gamma protein is a target of BAG3 regulatory activity in human tumor growth. Proc Natl Acad Sci USA 107: 7497-7502, 2010.

27 Lee YD, Cui MN, Yoon HH, Kim HY, Oh IH and Lee JH: Down-modulation of Bis reduces the invasive ability of glioma cells induced by TPA, through NF-kappaB mediated activation of MMP-9. BMB Rep 47: 262-267, 2014.

28 Kassis JN, Guancial EA, Doong H, Virador V and Kohn EC: CAIR-1/BAG-3 modulates cell adhesion and migration by downregulating activity of focal adhesion proteins. Exp Cell Res 312: 2962-2971, 2006.

29 Song F, Wang G, Ma Z, Ma Y and Wang Y: Silencing of BAG3 inhibits the epithelial-mesenchymal transition in human cervical cancer. Oncotarget 8: 95392-95400, 2017.

30 Li N, Du ZX, Zong ZH, Liu BQ, Li C, Zhang Q and Wang HQ: PKCdelta-mediated phosphorylation of BAG3 at Ser187 site induces epithelial-mesenchymal transition and enhances invasiveness in thyroid cancer FRO cells. Oncogene 32: 4539$4548,2013$. 
31 Zhao Q, Yue J, Zhang C, Gu X, Chen $\mathrm{H}$ and Xu L: Inactivation of M2 AChR/NF-kappaB signaling axis reverses epithelialmesenchymal transition (EMT) and suppresses migration and invasion in non-small cell lung cancer (NSCLC). Oncotarget 6: 29335-29346, 2015.

32 Sun Q, Yao X, Ning Y, Zhang W, Zhou G and Dong Y: Overexpression of response gene to complement 32 (RGC32) promotes cell invasion and induces epithelial-mesenchymal transition in lung cancer cells via the NF-kappaB signaling pathway. Tumour Biol 34: 2995-3002, 2013.

$33 \mathrm{Li} \mathrm{X,} \mathrm{Jiang} \mathrm{Z,} \mathrm{Li} \mathrm{X} \mathrm{and} \mathrm{Zhang} \mathrm{X:} \mathrm{SIRT1} \mathrm{overexpression} \mathrm{protects}$ non-small cell lung cancer cells against osteopontin-induced epithelial-mesenchymal transition by suppressing NF-kappaB signaling. Onco Targets Ther 11: 1157-1171, 2018.

34 Boiani M, Daniel C, Liu X, Hogarty MD and Marnett LJ: The stress protein BAG3 stabilizes Mcl-1 protein and promotes survival of cancer cells and resistance to antagonist ABT-737. J Biol Chem 288: 6980-6990, 2013.
35 Tanaka T, Shibazaki A, Ono R and Kaisho T: HSP70 mediates degradation of the p65 subunit of nuclear factor kappaB to inhibit inflammatory signaling. Sci Signal 7: ra119, 2014.

36 Wang $\mathrm{CH}$, Chou PC, Chung FT, Lin HC, Huang KH and Kuo HP: Heat shock protein 70 is implicated in modulating NF-kappa $B$ activation in alveolar macrophages of patients with active pulmonary tuberculosis. Sci Rep 7: 1214, 2017.

37 Rosati A, Leone A, Del Valle L, Amini S, Khalili K and Turco MC: Evidence for BAG3 modulation of HIV-1 gene transcription. J Cell Physiol 210: 676-683, 2007.

38 Cui MN, Yun HH, Lee NE, Kim HY, Im CN, Kim YS and Lee JH: Depletion of BIS sensitizes A549 cells to treatment with cisplatin. Mol Cell Toxicol 12: 63-71, 2016.

Received June 30, 2018

Revised July 11, 2018

Accepted July 12, 2018 